\title{
ON THE SPLINE-BASED WAVELET DIFFERENTIATION MATRIX
}

\author{
Leland Jameson ${ }^{1}$ \\ Institute for Computer Applications in Science and Engineering \\ NASA Langley Research Center \\ Hampton, VA 23681
}

\begin{abstract}
The differentiation matrix for a spline-based wavelet basis will be constructed. Given an $n$-th order spline basis it will be proven that the differentiation matrix is accurate of order $2 n+2$ when periodic boundary conditions are assumed. This high accuracy, or superconvergence, is lost when the boundary conditions are no longer periodic. Furthermore, it will be shown that spline-based bases generate a class of compact finite difference schemes.
\end{abstract}

\footnotetext{
${ }^{1}$ This research was supported by the National Aeronautics and Space Administration under NASA Contract No. NAS1-19480 while the author was in residence at the Institute for Computer Applications in Science and Engineering (ICASE), NASA Langley Research Center, Hampton, VA 23681. Research was also supported by AFOSR grant 93-1-0090, by DARPA grant N00014-91-4016, and by NSF grant DMS-9211820.
} 



\section{Introduction}

The use of wavelets as a basis set for the numerical solution of partial differential equations (PDE's) is a very active area of research. Wavelets, Daubechies or Splinebased, provide a very convenient structure for dividing information according to the frequency of the information at a given location. This splitting of the data by projecting onto a wavelet basis seems ideal for the solution of nonlinear PDE's where shocks or high frequencies might exist in a small portion of the domain, whereas in the remainder of the domain the solution might be comprised of very low frequency information.

In this paper the goal is to understand the character of a spline-based wavelet numerical method. Numerical results for such a method can be found in [12] and [6]. This understanding will be found through the differentiation matrix. In [4] the differentiation matrix was found for Daubechies wavelets with periodic boundary conditions, and a very high degree of differentiation accuracy known as superconvergence was proven to exist. Furthermore, it was seen that a Daubechies-based wavelet method corresponds to a finite difference method with grid refinement in regions where small scale data is present. In the more complicated scenario where periodic boundary conditions are no longer assumed, it was seen in [5] that the superconvergence is lost.

In this paper the differentiation matrix for a spline-based wavelet basis with periodic and nonperiodic boundary conditions will be constructed. It will be proven that a spline-based wavelet basis, also, displays superconvergence when periodic boundary conditions are assumed. Furthermore, the superconvergence is lost when periodicity is no longer assumed. In addition, it will be seen that spline-based wavelet methods generate a class of compact finite difference schemes.

Recall that the differentiation matrix is comprised of three matrices, $\mathcal{D}=\hat{C} D C$ :

- The first matrix $C$ is the quadrature matrix which maps from point values of a 
function to the approximate scaling function coefficients at the finest scale.

- The second matrix $D$ maps from the scaling function coefficients of a function to the scaling function coefficients of the derivative of the same function. For convenience, I will henceforth refer to this matrix as the 'derivative projection' matrix.

- In this paper the third matrix is the inverse of the first matrix: $\hat{C}=C^{-1}$.

This paper is organized as follows:

\section{Introduction}

\section{Definitions and Framework:}

Aliasing is reviewed.

\section{Multiple Scale Wavelet Decompositions:}

Wavelet methods use information at many scales, but all properties of waveletbased differentiation are dictated by differentiation in the finest scale approximation subspace, $V_{0}$.

\section{Differentiation in a Wavelet Subspace:}

Here we begin to develop the relations needed to build the differentiation matrix.

\section{Theoretical Accuracy:}

In this section the theoretical accuracy will be proved. For a spline-based wavelet basis using an $\mathbf{n}$-th order spline and assuming periodic boundary conditions, differentiation is accurate to order $2 n+2$.

\section{Equations for Finite Dimensions:}

In finite dimensions all wavelet operations can be written in matrix form. In this section, the matrix equations will be derived which will hold for arbitrary boundary conditions. 


\section{Examples with Periodic Boundary Conditions:}

Explicit differentiation matrices for the $n=1$ and $n=3$ spline-based wavelets will be given for periodic boundary conditions.

\section{Examples with Nonperiodic Boundary Conditions:}

Again, explicit examples are given. The most important point to note in this section is the loss of superconvergence at the boundaries.

\section{Loss of Superconvergence:}

The very important issue of the loss of superconvergence when nonperiodic boundary conditions are imposed is explored.

\section{Conclusion:}

The role of aliasing is noted in determining the order of accuracy of $\mathcal{D}$. It was shown in [4] that Daubechies-based wavelet methods have a strong relationship with ordinary finite difference methods. In this paper, a strong relationship between spline-based wavelet methods and compact finite difference schemes has been exposed. Furthermore, superconvergence is lost when boundary conditions other than periodic boundary conditions are imposed. 


\section{Definitions and Framework}

Please note that the framework, definitions, and even much of the notation in this paper come from a paper by M. Unser and A. Aldroubi, see [10].

\section{$2.1 \quad$ Fourier Transforms}

Let us set once and for all the definitions of the Fourier transform and the semidiscrete Fourier transform used in this paper. The Fourier transform of $f(x) \in L^{2}(R)$ is,

$$
\hat{f}(\xi)=\int_{-\infty}^{\infty} f(x) e^{i \xi x} d x
$$

and its inverse is,

$$
f(x)=\frac{1}{2 \pi} \int_{-\infty}^{\infty} \hat{f}(\xi) e^{-i \xi x} d \xi .
$$

The semi-discrete Fourier transform (SDFT) of $f_{k} \in l^{2}(Z)$ is,

$$
\hat{f}(\xi)=\sum_{k=-\infty}^{\infty} e^{i \xi k} f_{k}
$$

and its inverse is,

$$
f_{k}=\frac{1}{2 \pi} \int_{-\pi}^{\pi} \hat{f}(\xi) e^{-i \xi k} d \xi .
$$

\section{$2.2 \quad$ B-Splines}

All B-splines in this paper are central B-splines: centered at 0. The B-spline of order $n$ is denoted by $\beta^{n}(x)$. All splines of order $n$, denoted by $s^{n}(x)$, are a linear combination of B-splines of order $n$, see [7] and [8]:

$$
s^{n}(x)=\sum_{k} c_{k} \beta^{n}(x-k),
$$

and the space spanned by splines of order $n$ is denoted by $S^{n}$. The B-spline $\beta^{0}(x)$ is the box function, which is 1 for $x \in[-1 / 2,1 / 2]$ and zero otherwise. All B-splines of higher order are generated from $\beta^{0}(x)$ :

$$
\beta^{n}(x)=\beta^{0} * \beta^{n-1}(x),
$$


where ' $*$ ' denotes the convolution operator. Other useful forms of this equation are,

$$
\beta^{2 n+1}(x)=\beta^{n} * \beta^{n}(x),
$$

and

$$
\beta^{2 n+1}(x)=\int_{-\infty}^{\infty} \beta^{n}(y) \beta^{n}(x-y) d y
$$

and finally,

$$
\beta^{2 n+1}(x)=<\beta^{n}, \beta^{n}(x-\cdot)>
$$

where for $f, g \in L^{2}(R)$,

$$
<f, g>=\int_{-\infty}^{\infty} f(x) g(x) d x
$$

We will, also, need the samples of $\mathrm{B}$-splines at the integers:

$$
b^{n}(k)=\left.\beta^{n}(x)\right|_{x=k} .
$$

In order to use B-splines to construct a multiresolution analysis it is necessary that contractions and expansions of $n$-th order B-splines also be contained in $S^{n}$. This is only true when $n$ is odd, which is, therefore, assumed for this paper.

\subsection{Fourier Transforms of B-Splines}

From equation (6) we see that the Fourier transforms of B-splines are generated recursively. That is, the Fourier transform of a convolution is the product of the Fonrier transforms of the convolved functions, Therefore, the Fonrier transform of a B-spline is,

$$
\hat{\beta}^{n}(\xi)=\operatorname{sinc}^{n+1}(\xi)
$$

where $\sin e(\xi) \equiv \frac{\sin (\xi)}{\xi}$ and is the Fourier transform of $\beta^{\circ}(x)$.

\subsection{Aliasing and Fourier Transforms}

The relationship between the Fonrier transform and the semi-discrete Fourier transform and the role of aliasing is at the heart of a proof that will be given later in this 
paper. Therefore, I will reconstruct the straightforward algebra leading to aliasing using notation defined above.

Let $b_{k}^{n}$ be the samples of $\beta^{n}(x)$ as defined above:

$$
b_{k}^{n}=\beta^{n}(k)=\frac{1}{2 \pi} \int_{-\infty}^{\infty} \hat{\beta}^{n}(\xi) e^{-i \xi k} d \xi .
$$

Break the integral up as follows:

$$
b_{k}^{n}=\frac{1}{2 \pi} \sum_{r=-\infty}^{\infty} \int_{(2 r-1) \pi}^{(2 r+1) \pi} \hat{\beta}^{n}(\xi) e^{-i \xi k} d \xi
$$

which is equivalent to,

$$
b_{k}^{n}=\frac{1}{2 \pi} \sum_{r=-\infty}^{\infty} \int_{-\pi}^{\pi} \hat{\beta}^{n}(\xi+2 \pi r) e^{-i \xi k} d \xi
$$

where I have used the fact that $1=e^{-i 2 \pi r k}$. If we compare this expression with definition (4) we see that,

$$
\hat{b}^{n}(\xi)=\sum_{r=-\infty}^{\infty} \hat{\beta}^{n}(\xi+2 \pi r)
$$

which illustrates the aliasing of frequencies $\xi+2 \pi r$ onto frequency $\xi$.

\subsection{Spline-Based Wavelet Bases}

As mentioned above, the subspace of $\mathrm{n}$-th order splines, $S^{n}$, is spanned by $\mathrm{n}$-th order $\mathrm{B}$-splines. The following theorem will define the scaling functions for a spline-based wavelet basis, see [11]:

Theorem: The set of functions $\left\{\phi^{n}(x-k): k \in Z\right\}$ with

$$
\phi^{n}(x)=\sum_{k=-\infty}^{\infty} p_{k} \beta^{n}(x-k)
$$

is a basis of $S^{n}$ provided that the sequence $\{p\}$ is an invertible convolution operator from $l_{2}$ into itself.

The sequence $\{p\}$ is chosen such that the scaling function satisfies whatever properties are specified. That is, one can specify that the scaling functions and wavelets be orthogonal which places restrictions on $\{p\}$. There are many ways to define the 
scaling function, but in this paper it will be seen that in the calculation of the differentiation matrix that the sequence $\{p\}$ will 'divide out' producing a result which holds for all spline-based wavelet bases produced using the current framework.

Throughout the paper I will use the notation $\phi_{k}^{n}(x)$ to denote translation of $\phi^{n}(x)$ by $k$ :

$$
\phi_{k}^{n}(x)=\phi^{n}(x-k)
$$

Define the dual of $\phi^{n}, \tilde{\phi}^{n}$, as

$$
\tilde{\phi}^{n}(x)=\sum_{k=-\infty}^{\infty} r_{k} \beta^{n}(x-k),
$$

where the sequence $\{r\}$ is chosen such that

$$
\delta_{k}=<\tilde{\phi}_{0}, \phi_{k}>
$$

Later the relationship between $\{r\}$ and $\{p\}$ will be made precise. However, as mentioned above, $\{p\}$ and $\{r\}$ will have no affect on the differentiation matrix.

The wavelet is defined as,

$$
\psi^{n}(x)=\sum_{k=-\infty}^{\infty} q_{k} \bar{\beta}^{n}(x-k),
$$

and its dual is

$$
\tilde{\psi}^{n}(x)=\sum_{k=-\infty}^{\infty} s_{k} \bar{\beta}^{n}(x-k),
$$

where $\bar{\beta}$ is the 'B-spline wavelet'. For this paper this B-spline wavelet is not needed and will not be dealt with, see [10]. Furthermore, as with the scaling function, the sequences $\{q\}$ and $\{s\}$ defining the wavelet and its dual will be of no consequence in the calculation of the differentiation matrix.

This completes the definitions and framework. 


\section{Multiple Scale Wavelet Decompositions}

One of the underlying strengths of a wavelet decomposition is that it is possible to easily decompose a function, or signal, into its components at various scales. To illustrate, suppose that we are restricted to a finite number of dimensions, say $d$. Denote the finest scale approximation subspace by $V_{0}$, and the coefficients of the expansion in $V_{0}$ by $\vec{s}$. A usual wavelet decomposition would appear as,

$$
V_{0}=W_{1} \oplus W_{2} \oplus \ldots \oplus W_{J} \oplus V_{J}
$$

where, as is customary, $W_{j}$ denotes the wavelet subspace at scale $j$. A function projected onto the above subspaces would, therefore, be represented by $d$ coefficients, say $\vec{c}$. Under the assumption of finite dimensionality, there will be an invertible matrix mapping from $\vec{s}$ to $\vec{c}$ :

$$
\vec{c}=P \vec{s} .
$$

That is, if $g(x) \in V_{0}$ with the expansion,

$$
g(x)=\sum_{i=0}^{d-1} s_{i}^{0} \phi_{i}^{0}(x)
$$

and one projects into $V_{1}$ and $W_{1}$ by,

$$
P_{V_{1}} g(x)=\sum_{j=0}^{d / 2-1} \sum_{i=0}^{d-1} s_{i}^{0}<\phi_{i}^{0}, \tilde{\phi}_{j}^{1}>\phi_{j}^{1}(x),
$$

and

$$
P_{W_{1}} g(x)=\sum_{j=0}^{d / 2-1} \sum_{i=0}^{d-1} s_{i}^{0}<\phi_{i}^{0}, \tilde{\psi}_{j}^{1}>\psi_{j}^{1}(x),
$$

then the matrix form of the above linear transformations is denoted $P$ :

$$
P_{j, i}=<\phi_{i}^{0}, \tilde{\phi}_{j}^{1}>
$$

and

$$
P_{d / 2+j, i}=<\phi_{i}^{0}, \tilde{\psi}_{j}^{1}>
$$

for $i=0, \ldots, d-1$ and $j=0, \ldots, d / 2-1$. 
Suppose, now, that we have found the matrix $D$ mapping from $\vec{s}$ to $\vec{s}$, where $\vec{s}$ represents the coefficients of the approximation in $V_{0}$ of the derivative of a function. Of course, no one would choose to work with wavelets and stop the decomposition in the subspace $V_{0}$. The matrix $D$ does, however, characterize differentiation in any combination of wavelet subspaces, and the coefficients $\vec{c}$ can be found from,

$$
\vec{c}=P D P^{-1} \vec{c}
$$

To restate, $D$ dictates the character of wavelet differentiation. The primary concern in this paper is to understand $D$. Therefore, the remainder of this paper will not mention wavelet decompositions at multiple scales. 


\section{Theoretical Accuracy}

The accuracy of the spline based differentiation matrix can be proved by observing the behavior of the semi-discrete Fourier transform of

$$
d_{q}=<\tilde{\phi}_{0}, \dot{\phi}_{q}>
$$

near $\xi=0$. It will be shown that the Taylor series of $\hat{d}(\xi)$ about $\xi=0$ is of the form,

$$
\hat{d}(\xi)=i \xi+O\left(\xi^{2 n+3}\right)+\ldots
$$

and this implies accuracy of order $2 n+2$.

\subsection{The Semi-Discrete Fourier Transform of $\{d\}$}

As above, define $d_{q}$ as,

$$
d_{q}=<\tilde{\phi}_{0}, \dot{\phi}_{q}>
$$

and recall from the previous section that this is,

$$
d_{q}=\sum_{l, m} p_{l} r_{m} \int \beta^{n}(x-m) \dot{\beta}^{n}(x-l-q) d x .
$$

Again, note that as of now all summations and integrals are from $-\infty$ to $\infty$ allowing one to shift summations and integrals without changing the limits,

$$
d_{q}=\sum_{l, m} p_{l} r_{m} \int \beta^{n}(x) \dot{\beta}^{n}(x+m-l-q) d x .
$$

Let us rename this integral as,

$$
a_{i+q-m}=\int \beta^{n}(x) \dot{\beta}^{n}(x+m-l-q) d x
$$

which yields the following expression for $d_{q}$,

$$
d_{q}=\sum_{l, m} p_{l} r_{m} a_{l+q-m}
$$

Break apart the double summation,

$$
d_{q}=\sum_{l} p_{l} \sum_{m} r_{m} a_{l+q-m}
$$


and shift the index $l$,

$$
d_{q}=\sum_{l} p_{l-q} \sum_{m} r_{m} a_{l-m} .
$$

Let the superscript " " " denote time reversal of a sequence to get,

$$
d_{q}=\sum_{l} p_{q-l}^{\prime} \sum_{m} r_{m} a_{l-m}
$$

We see that the right-hand side is a double convolution. We can, therefore, use the convolution theorem to get,

$$
\hat{d}(\xi)=\hat{p}^{\prime}(\xi) \hat{r}(\xi) \hat{a}(\xi)
$$

Recall from the previous section that the following expression for $\hat{r}(\xi)$ was found:

$$
\hat{r}(\xi)=\left(\hat{p}^{\prime}(\xi) \hat{b}^{2 n+1}(\xi)\right)^{-1}
$$

Using this expression for $\hat{r}(\xi)$ in the expression for $\hat{d}(\xi)$ we get,

$$
\hat{d}(\xi)=\frac{\hat{a}(\xi)}{\hat{b}^{2 n+1}(\xi)} .
$$

The next two subsections wil derive $\hat{a}(\xi)$ and $\hat{b}(\xi)$. After these two expressions are found the proof of accuracy will begin.

\subsection{The Semi-Discrete Fourier Transform of $\{a\}$}

Define the projection of the derivative of a B-spline onto a B-spline as,

$$
\alpha(x)=\int_{-\infty}^{\infty} \beta^{n}(y) \hat{\beta}^{n}(y-x) d y
$$

Recalling that,

$$
\hat{f}(\xi)=i \xi \hat{f}(\xi)
$$

and from the convolution theorem, see the introduction, we get the Fourier transform of $\alpha(x)$,

$$
\hat{\alpha}(\xi)=i \xi \sin c^{2 n+2}(\xi) .
$$


The sequence $a_{p}$ is defined as the samples of $\alpha(x)$ at the integers,

$$
a_{p}=\int \beta^{n}(x) \dot{\beta}^{n}(x-p) d x .
$$

The semi-discrete Fourier transform of $a_{p}$ is, due to aliasing (see the introduction),

$$
\hat{a}(\xi)=i \sum_{r=-\infty}^{\infty}(\xi+2 \pi r) \sin c^{2 n+2}(\xi+2 \pi r) .
$$

\subsection{The Semi-Discrete Fourier Transform of $\{b\}$}

Recall that the samples of the B-spline $\beta^{2 n+1}(x)$ are found from,

$$
b_{k}^{2 n+1}=\int \beta^{n}(x) \beta^{n}(x-k) d x .
$$

We know the Fourier transform of $\beta^{2 n+1}(x)$ from the introduction:

$$
\hat{\beta}^{2 n+1}(\xi)=\operatorname{sinc}^{2 n+2}(\xi) .
$$

Also, from the introduction we know how to get the semi-discrete Fourier transform of $b_{k}^{2 n+1}$ from $\hat{\beta}^{2 n+1}(\xi)$,

$$
\hat{b}^{2 n+1}(\xi)=\sum_{q=-\infty}^{\infty} \operatorname{sinc}^{2 n+2}(\xi+2 \pi q) .
$$

The semi-discrete Fourier transforms $\hat{a}(\xi)$ and $\hat{b}(\xi)$ will now be used in the proof of accuracy.

\subsection{Proof of Accuracy}

The proof of superconvergence begins by first proving two lemmas.

Lemma A: The series,

$$
s_{1}(\xi)=\sum_{q=-\infty}^{\infty} \operatorname{sinc}^{2 n+2}(\xi+2 \pi q),
$$

is an even function of $\xi$ and has a Taylor series about $\xi=0$ of the form,

$$
s_{1}(\xi)=1+c_{1} \xi^{2}+c_{2} \xi^{4}+\ldots
$$


Proof: Using the definition of a sinc function we get,

$$
s_{1}(\xi)=\sum_{q=-\infty}^{\infty} \frac{\sin ^{2 n+2}(\xi+2 \pi q)}{(\xi+2 \pi q)^{2 n+2}} .
$$

We can simplify the numerator $\operatorname{since} \sin (\xi)$ is periodic with period $2 \pi$,

$$
s_{1}(\xi)=\sum_{q=-\infty}^{\infty} \frac{\sin ^{2 n+2}(\xi)}{(\xi+2 \pi q)^{2 n+2}} .
$$

To show that $s_{1}(\xi)$ is even first let $q=0$ to get,

$$
\frac{\sin ^{2 n+2}(\xi)}{\xi^{2 n+2}}=\left(1-1 / 6 \xi^{2}+1 / 120 \xi^{4}+\ldots\right)^{2 n+2},
$$

which is even. Now, sum the terms for $q=-1$ and $q=1$,

$$
g(\xi)=\frac{\sin ^{2 n+2}(\xi)}{(\xi+2 \pi)^{2 n+2}}+\frac{\sin ^{2 n+2}(\xi)}{(\xi-2 \pi)^{2 n+2}}
$$

to get,

$$
g(\xi)=g(-\xi)
$$

Matching every term with positive $q$ with every term with negative $q$ we see that $s_{1}(\xi)$ is an even function.

We can see from equation (55) that the first few terms in the Taylor series when $q=0$ are $1+c_{1} \xi^{2}+c_{2} \xi^{4}+\ldots$. On the other hand, when $q \neq 0$ each term in the summation is of the form,

$$
P(\xi)=\frac{\sin ^{2 n+2}(\xi)}{(\xi+2 \pi q)^{2 n+2}}
$$

or

$$
(\xi+2 \pi q)^{2 n+2} P(\xi)=\sin ^{2 n+2}(\xi)
$$

Using the series expansions of these expressions we get,

$$
\left(\alpha_{1}+\alpha_{2} \xi+\alpha_{3} \xi^{2}+\ldots\right) P(\xi)=\left(\xi-\xi^{3} / 6+\xi^{5} / 120+\ldots\right)^{2 n+2}
$$

or

$$
\left(\alpha_{1}+\alpha_{2} \xi+\alpha_{3} \xi^{2}+\ldots\right) P(\xi)=\xi^{2 n+2}+\text { h.o.t. }
$$


This implies that,

$$
P(\xi)=O\left(\xi^{2 n+2}\right)+\text { h.o.t. }
$$

That is, all terms in $s_{1}(\xi)$ with $q \neq 0$ contribute terms $O\left(\xi^{2 n+2}\right)$, and therefore, all low order terms come from equation (55). This yields the desired result. //

\section{Lemma B:}

The series,

$$
s_{2}(\xi)=\sum_{r=-\infty}^{\infty} r \sin c^{2 n+2}(\xi+2 \pi r),
$$

is an odd function of $\xi$ and has a Taylor series about $\xi=0$ of the form,

$$
s_{2}(\xi)=\alpha_{1} \xi^{2 n+3}+\ldots
$$

Proof: First of all, due to the $2 \pi$ periodicity of $\sin (\xi), s_{2}(\xi)$ becomes,

$$
s_{2}(\xi)=\sum_{r=-\infty}^{\infty} r \frac{\sin ^{2 n+2}(\xi)}{(\xi+2 \pi r)^{2 n+2}} .
$$

Note that the $r=0$ term in this sum makes no contribution to $s_{2}(\xi)$. Now, note that each term in the sum has the following form,

$$
f(\xi, r)=r \frac{\sin ^{2 n+2}(\xi)}{(\xi+2 \pi r)^{2 n+2}},
$$

and, consequently, the function $g(\xi, r)$ defined as follows is an odd function of $\xi$ :

$$
g(\xi, r)=f(\xi, r)+f(\xi,-r)=-(f(-\xi, r)+f(-\xi,-r))
$$

This implies that $s_{2}(\xi)$ is an odd function of $\xi$ since,

$$
s_{2}(\xi)=\sum_{r=1}^{\infty} g(\xi, r) .
$$


Therefore, the Taylor series of $s_{2}(\xi)$ about $\xi=0$ must contain only odd powers of $\xi$.

Consider, now, a typical term of $s_{2}(\xi)$ for $r \neq 0$,

$$
P(\xi)=\frac{\sin ^{2 n+2}(\xi)}{(\xi+2 \pi r)^{2 n+2}} .
$$

Rewrite this to get,

$$
(\xi+2 \pi r)^{2 n+2} P(\xi)=\sin ^{2 n+2}(\xi) .
$$

Note that the binomial on the left-hand side contains all powers of $\xi$, and that $\sin ^{2 n+2}(\xi)$ contains only even powers of $\xi$ starting with the term $\xi^{2 n+2}$ :

$$
\left(c_{0}+c_{1} \xi+c_{2} \xi^{2}+\ldots\right) P(\xi)=\xi^{2 n+2}+d_{1} \xi^{2 n+4}+\ldots
$$

We see now that the first term of $P(\xi)$ must be $\xi^{2 n+2} / c_{0}$, but this term will also multiply the $c_{1} \xi$ term in the binomial expression requiring that $P(\xi)$ have as a second term $\alpha_{1} \xi^{2 n+3}$ where $\alpha_{1}=-c_{1} /\left(c_{0}^{2}\right)$. The point is that there exist a nonzero term with power $2 n+3$ in the polynomial $P(\xi)$.

That is, in general the series expansion of the function $f(\xi, r)$ about $\xi=0$ contains all powers $p$ of $\xi$ with $p \geq 2 n+2$, and when added to $f(\xi,-r)$ the even powers of $\xi$ must cancel leaving as the first term the term with the power $2 n+3$. That is,

$$
s_{2}(\xi)=\alpha \xi^{2 n+3}+\ldots
$$

This is the desired result. //

Theorem: The semi-discrete Fourier transform of the sequence $d_{k}$ has a series expansion about $\xi=0$ of the following form:

$$
\hat{d}(\xi)=i \xi+O\left(\xi^{2 n+3}\right)+\ldots
$$

Proof: Recall that the semi-discrete Fourier transform of $\{d\}$ is,

$$
\hat{d}(\xi)=\frac{\hat{a}(\xi)}{\hat{b}^{2 n+2}(\xi)} .
$$


Now, substitute in the previously derived expressions for $\hat{a}(\xi)$ and $\hat{b}^{2 n+2}(\xi)$ to get,

$$
\hat{d}(\xi)=\frac{i \sum_{r=-\infty}^{\infty}(\xi+2 \pi r) \sin c^{2 n+2}(\xi+2 \pi r)}{\sum_{q=-\infty}^{\infty} \sin c^{2 n+2}(\xi+2 \pi q)}
$$

Multiply both sides by the denominator to get,

$$
\hat{d}(\xi) \sum_{q=-\infty}^{\infty} \sin c^{2 n+2}(\xi+2 \pi q)=i \sum_{r=-\infty}^{\infty}(\xi+2 \pi r) \sin c^{2 n+2}(\xi+2 \pi r)
$$

and combine similar terms to get,

$$
(\hat{d}(\xi)-i \xi) \cdot \sum_{q=-\infty}^{\infty} \sin c^{2 n+2}(\xi+2 \pi q)=2 \pi \sum_{r=-\infty}^{\infty} r \sin c^{2 n+2}(\xi+2 \pi r)
$$

Now, apply the results from Lemmas $\mathrm{A}$ and $\mathrm{B}$ to get,

$$
(\hat{d}(\xi)-i \xi)\left(1+c_{1} \xi^{2}+\ldots\right)=\alpha \xi^{2 n+3}+\ldots
$$

This equation implies the desired result,

$$
\hat{d}(\xi)=i \xi+O\left(\xi^{2 n+3}\right)+\ldots
$$

This completes the proof. //

This proves that the sequence $\{d\}$ differentiates with accuracy $2 n+2$, and, consequently, the differentiation matrix containing the periodic version of $\{d\}$ as its rows will differentiate with accuracy $2 n+2$.

The next section will provide explicit examples of the differentiation matrices for the first and third order B-splines. Also, the accuracy will be shown computationally. 


\section{Equations for Finite Dimensions}

The equations that will be derived in this section will hold for any spline-based wavelet basis defined on a finite-dimensional domain. This includes the equations for both periodic and nonperiodic boundary conditions. Note that in this section all summations are from 0 to $d-1$, and consequently the summation limits will often not be shown.

\subsection{Quadrature}

Let $g(x)$ denote the projection of $f(x) \in L^{2}(R)$ in $V_{0}$ :

$$
g(x)=P_{V_{0}} f(x)=\sum_{l=0}^{d-1} s_{l} \phi_{l}(x),
$$

where

$$
s_{l}=<f, \tilde{\phi}_{l}>
$$

Note that in this section the notation $b_{m}(x)$ will be used to denote the B-spline basis functions. For periodic boundary conditions one could have simply used $\beta(x-$ $m$ ), but for nonperiodic boundary conditions the basis functions near the boundary are truncated B-splines. The notation used here is, therefore, general enough for both periodic and nonperiodic problems. That is, for a nonperiodic domain $b_{0}(x)$ will be only the right-hand portion with support width 1 of the $B$-spline being used. Now, using the B-spline expansion of the scaling function in equation (79) we get,

$$
g(x)=\sum_{l} s_{l} \sum_{m} p_{l, m} b_{m}(x) .
$$

Combine the summations,

$$
g(x)=\sum_{l, m} s_{l} p_{l, m} b_{m}(x),
$$

and sample $g(x)$ at the integers to get,

$$
g(k)=\sum_{l, m} s_{l} p_{l, m} b_{m}(k),
$$


or simply,

$$
g_{k}=\sum_{l, m} s_{l} p_{l, m} b_{m, k}
$$

Group the terms as,

$$
g_{k}=\sum_{l}\left[\sum_{m} p_{l, m} b_{m, k}\right] s_{l}
$$

and rename the inner group as $\gamma$ to get,

$$
g_{k}=\sum_{l} \gamma_{l, k} s_{l}
$$

Now, transpose $\gamma$,

$$
g_{k}=\sum_{l} \gamma_{k, l}^{T} s_{l}
$$

and utilize matrix and vector notation to get,

$$
\vec{g}=(P b)^{T} \vec{s}
$$

This expression is a relationship between the samples of $g(x) \in V_{0}$ and the exact scaling function coefficients. In general we need a relationship between the samples of $f(x) \in L^{2}(R)$ and an approximate set of scaling function coefficients $\vec{\sigma}$,

$$
\vec{f}=(P b)^{T} \vec{\sigma}
$$

This completes the matrix form of the quadrature formula. The next subsection will derive the matrix form of the relationship between the scaling function and its dual.

\subsection{The Scaling Function and its Dual}

Recall once more the relationship between the scaling function and its dual,

$$
\delta_{i, j}=<\phi_{i}, \tilde{\phi}_{j}>
$$

In terms of the B-spline expansions we get,

$$
\delta_{i, j}=\sum_{m} \sum_{l} p_{i, m} r_{j, l} \int b_{m}(x) b_{l}(x) d x
$$


Let $B$ denote the matrix form of the above integral. That is, $B$ contains the projections of the B-splines projected onto other B-splines. The above equation becomes,

$$
\delta_{i, j}=\sum_{l} r_{j, l}\left[\sum_{m} p_{i, m} B_{m, l}\right]
$$

Now, rename the expression in the brackets $\gamma$ to get,

$$
\delta_{i, j}=\sum_{l} r_{j, l} \gamma_{i, l}
$$

Transpose $r$ to get,

$$
\delta_{i, j}=\sum_{l} \gamma_{i, l} r_{l, j}^{T}
$$

The above equation has the following matrix form:

$$
I=P B R^{T}
$$

This is the matrix form of the requirement that the scaling function be orthogonal to its dual under translation. The next subsection will derive the derivative projection matrix.

\subsection{The Derivative Projection Matrix}

Recall from an earlier section that the elements of the derivative projection matrix come from the following inner product:

$$
d_{j, i}=<\dot{\phi}_{i}, \tilde{\phi}_{j}>
$$

Replace the scaling functions with their B-spline expansions to get,

$$
d_{j, i}=\sum_{m, l} p_{i, m} r_{j, l} \int \dot{b}_{m}(x) b_{l}(x) d x
$$

Let the matrix $A$ contain the results of the above integration,

$$
d_{j, i}=\sum_{m, l} p_{i, m} r_{j, l} A_{l, m}
$$

Now, group the terms as,

$$
d_{j, i}=\sum_{m} p_{i, m}\left[\sum_{l} r_{j, l} A_{l, m}\right]
$$


and rename the bracketed expression as $\gamma$ to get,

$$
d_{j, i}=\sum_{m} p_{i, m} \gamma_{j, m} .
$$

Transpose $p$ to get,

$$
d_{j, i}=\sum_{m} \gamma_{j, m} p_{m, i}^{T},
$$

and the matrix form of this equation is,

$$
D=R A P^{T} .
$$

This completes the derivation of the derivative projection matrix. The following subsection will combine the results from all the subsections in this section to get the matrix form of the differentiation matrix.

\subsection{The Differentiation Matrix}

The differentiation matrix is,

$$
\mathcal{D}=\hat{C} D \hat{C}^{-1},
$$

where

$$
\hat{C}=(P b)^{T} .
$$

From above we found that,

$$
D=R A P^{T},
$$

and

$$
I=P B R^{T} .
$$

From equation (106) we get,

$$
I=R B^{T} P^{T} .
$$

Solve for $R$ and use the fact the $B$ is symmetric to get,

$$
R=\left(B P^{T}\right)^{-1} .
$$


Use this expression in equation (105) to get,

$$
D=\left(P^{T}\right)^{-1} B^{-1} A P^{T} .
$$

Now, using the expression for the quadrature we get the differentiation matrix,

$$
\mathcal{D}=b^{T} P^{T}\left(P^{T}\right)^{-1} B^{-1} A P^{T}\left(P^{T}\right)^{-1}\left(b^{T}\right)^{-1},
$$

or

$$
\mathcal{D}=b^{T} B^{-1} A\left(b^{T}\right)^{-1} .
$$

Let me restate two notational definitions. First, the elements of the matrix ' $b$ ' are $b_{m, k}$ which denotes sampling basis ' $m$ ' at position ' $k$ '. Second, the elements of the matrix ' $B$ ' are $\int b_{m}(x) b_{k}(x) d x$.

Note that in the case of periodic boundary conditions that all three of the matrices $b, A$, and $B$ are circulant. They, therefore, commute yielding $B^{-1} A$ as the differentiation matrix. 


\section{Examples with Periodic Boundary Conditions}

In this section explicit examples for the 1st and 3rd order spline-based wavelet differentiation matrices will be given. First, the accuracy will be illustrated explicitly by calculating the semi-discrete Fourier transforms for the filters $\vec{a}, \vec{b}$, and $\vec{d}$. Then the matrix forms, $A, B$, and $D$ will be given.

\subsection{The Differentiation Matrix for a 1-st Order B-Spline}

The first order spline is the convolution of two box functions. It is defined as $\beta^{\mathbf{1}}(x)=$ $x+1$ for $x \in[-1,0], \beta^{1}(x)=1-x$ for $x \in(0,1]$ and 0 otherwise.

\subsubsection{The Filters $\vec{a}, \vec{b}$, and $\vec{d}$}

For the 1-st order B-spline the filters $\vec{a}$ and $\vec{b}$ are,

$$
\vec{a}=\{-1 / 2,0,1 / 2\},
$$

and

$$
\vec{b}^{3}=\{1 / 6,2 / 3,1 / 6\}
$$

The filter $\vec{d}$ is defined such that,

$$
b^{3} * d=a
$$

Therefore,

$$
\hat{d}(\xi)=\frac{\hat{a}(\xi)}{\hat{b}^{3}(\xi)}
$$

where,

$$
\hat{a}(\xi)=i \sin (\xi)
$$

and,

$$
\hat{b}^{3}(\xi)=1 / 3(2+\cos (\xi))
$$

$\hat{d}(\xi)$ will have the form,

$$
\hat{d}(\xi)=i \xi+c \xi^{q}+h o t
$$


and must satisfy

$$
\left(i \xi+c \xi^{q}\right) 1 / 3(2+\cos (\xi))=i \sin (\xi)
$$

Consider the first few terms of each series,

$$
\left(i \xi+c \xi^{q}\right) 1 / 3\left(2+1-\xi^{2} / 2+\xi^{4} / 24+h o t\right)=i\left(\xi-\xi^{3} / 6+\xi^{5} / 120+h o t\right)
$$

or

$$
\left(i \xi+c \xi^{q}\right)\left(1-\xi^{2} / 6+\xi^{4} / 72+h o t\right)=i\left(\xi-\xi^{3} / 6+\xi^{5} / 120+h o t\right)
$$

and finally,

$$
i\left(\xi-\xi^{3} / 6\right)+i \xi^{5} / 72+c \xi^{9}+h o t=i\left(\xi-\xi^{3} / 6\right)+i \xi^{5} / 120+h o t
$$

with the conclusion that

$$
q=5
$$

This is exactly the requirement for 4 -th order accuracy.

\subsubsection{The Differentiation Matrix}

In matrix form, the filter $\vec{a}$ is,

$$
A=\left[\begin{array}{cccccccccc}
0 & 1 / 2 & 0 & 0 & 0 & 0 & 0 & 0 & 0 & -1 / 2 \\
-1 / 2 & 0 & 1 / 2 & 0 & 0 & 0 & 0 & 0 & 0 & 0 \\
0 & -1 / 2 & 0 & 1 / 2 & 0 & 0 & 0 & 0 & 0 & 0 \\
0 & 0 & -1 / 2 & 0 & 1 / 2 & 0 & 0 & 0 & 0 & 0 \\
0 & 0 & 0 & -1 / 2 & 0 & 1 / 2 & 0 & 0 & 0 & 0 \\
0 & 0 & 0 & 0 & -1 / 2 & 0 & 1 / 2 & 0 & 0 & 0 \\
0 & 0 & 0 & 0 & 0 & -1 / 2 & 0 & 1 / 2 & 0 & 0 \\
0 & 0 & 0 & 0 & 0 & 0 & -1 / 2 & 0 & 1 / 2 & 0 \\
0 & 0 & 0 & 0 & 0 & 0 & 0 & -1 / 2 & 0 & 1 / 2 \\
1 / 2 & 0 & 0 & 0 & 0 & 0 & 0 & 0 & -1 / 2 & 0
\end{array}\right]
$$

and the filter $\vec{b}$ is,

$$
B=\left[\begin{array}{cccccccccc}
2 / 3 & 1 / 6 & 0 & 0 & 0 & 0 & 0 & 0 & 0 & 1 / 6 \\
1 / 6 & 2 / 3 & 1 / 6 & 0 & 0 & 0 & 0 & 0 & 0 & 0 \\
0 & 1 / 6 & 2 / 3 & 1 / 6 & 0 & 0 & 0 & 0 & 0 & 0 \\
0 & 0 & 1 / 6 & 2 / 3 & 1 / 6 & 0 & 0 & 0 & 0 & 0 \\
0 & 0 & 0 & 1 / 6 & 2 / 3 & 1 / 6 & 0 & 0 & 0 & 0 \\
0 & 0 & 0 & 0 & 1 / 6 & 2 / 3 & 1 / 6 & 0 & 0 & 0 \\
0 & 0 & 0 & 0 & 0 & 1 / 6 & 2 / 3 & 1 / 6 & 0 & 0 \\
0 & 0 & 0 & 0 & 0 & 0 & 1 / 6 & 2 / 3 & 1 / 6 & 0 \\
0 & 0 & 0 & 0 & 0 & 0 & 0 & 1 / 6 & 2 / 3 & 1 / 6 \\
1 / 6 & 0 & 0 & 0 & 0 & 0 & 0 & 0 & 1 / 6 & 2 / 3
\end{array}\right]
$$


The derivative projection matrix $D$, which recall is the same as the differentiation matrix $\mathcal{D}$ for periodic boundary conditions, is

$$
D=B^{-1} A .
$$

A 10 by 10 version of the derivative projection matrix for the current scenario is,

$$
D=\left[\begin{array}{cccccccccc}
0 & .804 & -.215 & .057 & -.014 & 0 & .014 & -.057 & .215 & -.804 \\
-.804 & 0 & .804 & -.215 & .057 & -.014 & 0 & .014 & -.057 & .215 \\
.215 & -.804 & 0 & .804 & -.215 & .057 & -.014 & 0 & .014 & -.057 \\
-.057 & .215 & -.804 & 0 & .804 & -.215 & .057 & -.014 & 0 & .014 \\
.014 & -.057 & .215 & -.804 & 0 & .804 & -.215 & .057 & -.014 & 0 \\
0 & .014 & -.057 & .215 & -.804 & 0 & .804 & -.215 & .057 & -.014 \\
-.014 & 0 & .014 & -.057 & .215 & -.804 & 0 & .804 & -.215 & .057 \\
.057 & -.014 & 0 & .014 & -.057 & .215 & -.804 & 0 & .804 & -.215 \\
-.215 & .057 & -.014 & 0 & .014 & -.057 & .215 & -.804 & 0 & .804 \\
.804 & -.215 & .057 & -.014 & 0 & .014 & -.057 & .215 & -.804 & 0
\end{array}\right]
$$

\subsection{The Differentiation Matrix for a 3-rd Order B-Spline}

The 3-rd order B-spline is the convolution of two 1-st order B-splines. The (nonnormalized) 3-rd order B-spline is defined as, $\beta^{3}(x)=(x+2)^{3}$ for $x \in[-2,-1)$, $\beta^{3}(x)=4-6 x^{2}-3 x^{3}$ for $x \in[-1,0), \beta^{3}(x)=4-6 x^{2}+3 x^{3}$ for $x \in[0,1)$, $\beta^{3}(x)=(2-x)^{3}$ for $x \in[1,2]$, and 0 otherwise.

\subsubsection{The Filters $\vec{a}, \vec{b}$, and $\vec{d}$}

The explicitly calculated filters for the 3-rd order B-spline are,

$$
\vec{a}=\{-1 / 20,-14 / 5,-49 / 4,0,49 / 4,14 / 5,1 / 20\},
$$

and

$$
\vec{b}^{7}=\{1 / 140,6 / 7,1191 / 140,604 / 35,1191 / 140,6 / 7,1 / 140\} .
$$

Normalization is not necessary since the filter $\vec{a}$ is divided by the filter $\vec{b}^{7}$. The semi-discrete Fourier transforms are,

$$
\hat{a}(\xi)=i(49 / 2 \sin (\xi)+28 / 5 \sin (2 \xi)+1 / 10 \sin (3 \xi)),
$$


and

$$
\hat{b}^{7}(\xi)=604 / 35+1191 / 70 \cos (\xi)+12 / 7 \cos (2 \xi)+1 / 70 \cos (3 \xi)
$$

Again, the semi-discrete Fourier transforms are related by,

$$
\hat{b}^{7}(\xi) \hat{d}(\xi)=\hat{a}(\xi)
$$

In a manner analogous to the previous subsection, we equate powers in the Taylor series of the semi-discrete Fourier transforms and come to the conclusion that,

$$
\hat{d}(\xi)=i \xi+c \xi^{9}+\text { hot }
$$

This is precisely the condition that is required for 8 -th order accuracy.

\subsubsection{The Differentiation Matrix}

The matrices for the filters $\vec{a}$ and $\vec{b}$ are,

$$
\begin{aligned}
& A= \\
& {\left[\begin{array}{cccccccccc}
0 & 49 / 4 & 14 / 5 & 1 / 20 & 0 & 0 & 0 & -1 / 20 & -14 / 5 & -49 / 4 \\
-49 / 4 & 0 & 49 / 4 & 14 / 5 & 1 / 20 & 0 & 0 & 0 & -1 / 20 & -14 / 5 \\
-14 / 5 & -49 / 4 & 0 & 49 / 4 & 14 / 5 & 1 / 20 & 0 & 0 & 0 & -1 / 20 \\
-1 / 20 & -14 / 5 & -49 / 4 & 0 & 49 / 4 & 14 / 5 & 1 / 20 & 0 & 0 & 0 \\
0 & -1 / 20 & -14 / 5 & -49 / 4 & 0 & 49 / 4 & 14 / 5 & 1 / 20 & 0 & 0 \\
0 & 0 & -1 / 20 & -14 / 5 & -49 / 4 & 0 & 49 / 4 & 14 / 5 & 1 / 20 & 0 \\
0 & 0 & 0 & -1 / 20 & -14 / 5 & -49 / 4 & 0 & 49 / 4 & 14 / 5 & 1 / 20 \\
1 / 20 & 0 & 0 & 0 & -1 / 20 & -14 / 5 & -49 / 4 & 0 & 49 / 4 & 14 / 5 \\
14 / 5 & 1 / 20 & 0 & 0 & 0 & -1 / 20 & -14 / 5 & -49 / 4 & 0 & 49 / 4 \\
49 / 4 & 14 / 5 & 1 / 20 & 0 & 0 & 0 & -1 / 20 & -14 / 5 & -49 / 4 & 0
\end{array}\right]}
\end{aligned}
$$

and

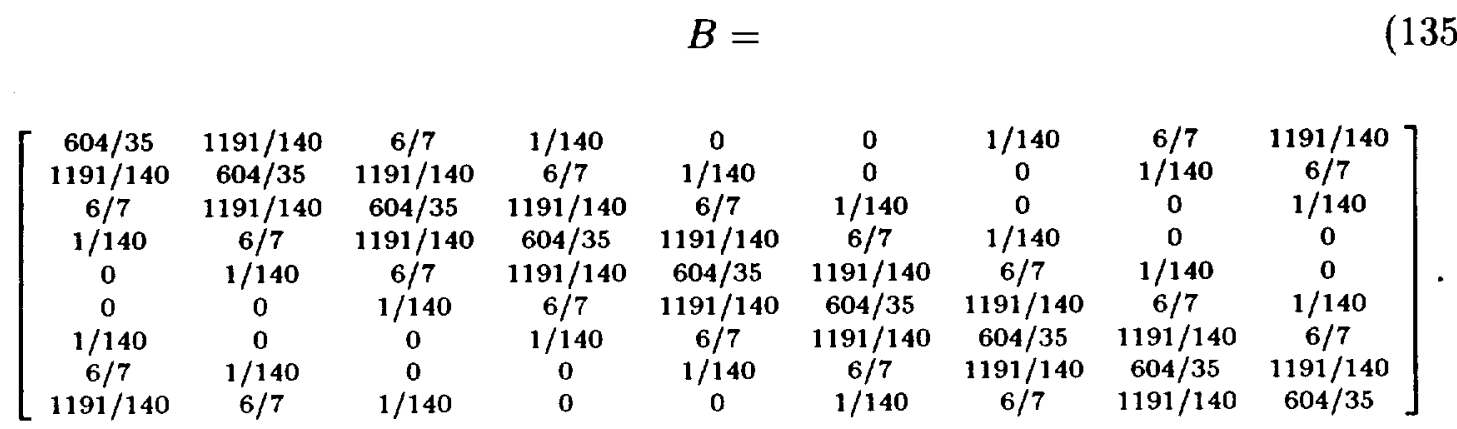


The derivative projection matrix is

$$
D=B^{-1} A,
$$

or,

$$
\begin{aligned}
& D= \\
& {\left[\begin{array}{cccccccccc}
0 & .938 & -.387 & .184 & -.078 & 0 & .078 & -.184 & .387 & -.938 \\
-.938 & 0 & .938 & -.387 & .184 & -.078 & 0 & .078 & -.184 & .387 \\
.387 & -.938 & 0 & .938 & -.387 & .184 & -.078 & 0 & .078 & -.184 \\
-.184 & .387 & -.938 & 0 & .938 & -.387 & .184 & -.078 & 0 & .078 \\
.078 & -.184 & .387 & -.938 & 0 & .938 & -.387 & .184 & -.078 & 0 \\
0 & .078 & -.184 & .387 & -.938 & 0 & .938 & -.387 & .184 & -.078 \\
-.078 & 0 & .078 & -.184 & .387 & -.938 & 0 & .938 & -.387 & .184 \\
.184 & -.078 & 0 & .078 & -.184 & .387 & -.938 & 0 & .938 & -.387 \\
-.387 & .184 & -.078 & 0 & .078 & -.184 & .387 & -.938 & 0 & .938 \\
.938 & -.387 & .184 & -.078 & 0 & .078 & -.184 & .387 & -.938 & 0
\end{array}\right]}
\end{aligned}
$$




\section{Nonperiodic Boundary Conditions}

In this section the differentiation matrix will be given for one particular boundary construction. The construction considered here is one of truncated B-splines. That is, away from the boundaries one simply shifts the B-splines by one to generate a spline basis. At the boundaries we simply continue this shifting. If part of the Bspline goes beyond the domain then simply truncate. This construction maintains the approximation properties of the spline subspace. That is, any $\mathbf{n}$-th order spline can be generated across the domain using $\mathrm{n}$-th order $\mathrm{B}$-splines truncated in this way.

\subsection{1st Order Spline}

Using the boundary construction outlined in the previous paragraph and the 1st order B-spline one gets the following matrices:

$$
A=\left[\begin{array}{cccccccccc}
-1 / 2 & 1 / 2 & 0 & 0 & 0 & 0 & 0 & 0 & 0 & 0 \\
-1 / 2 & 0 & 1 / 2 & 0 & 0 & 0 & 0 & 0 & 0 & 0 \\
0 & -1 / 2 & 0 & 1 / 2 & 0 & 0 & 0 & 0 & 0 & 0 \\
0 & 0 & -1 / 2 & 0 & 1 / 2 & 0 & 0 & 0 & 0 & 0 \\
0 & 0 & 0 & -1 / 2 & 0 & 1 / 2 & 0 & 0 & 0 & 0 \\
0 & 0 & 0 & 0 & -1 / 2 & 0 & 1 / 2 & 0 & 0 & 0 \\
0 & 0 & 0 & 0 & 0 & -1 / 2 & 0 & 1 / 2 & 0 & 0 \\
0 & 0 & 0 & 0 & 0 & 0 & -1 / 2 & 0 & 1 / 2 & 0 \\
0 & 0 & 0 & 0 & 0 & 0 & 0 & -1 / 2 & 0 & 1 / 2 \\
0 & 0 & 0 & 0 & 0 & 0 & 0 & 0 & -1 / 2 & 1 / 2
\end{array}\right]
$$

and the matrix $B$ is,

$$
B=\left[\begin{array}{cccccccccc}
1 / 3 & 1 / 6 & 0 & 0 & 0 & 0 & 0 & 0 & 0 & 0 \\
1 / 6 & 2 / 3 & 1 / 6 & 0 & 0 & 0 & 0 & 0 & 0 & 0 \\
0 & 1 / 6 & 2 / 3 & 1 / 6 & 0 & 0 & 0 & 0 & 0 & 0 \\
0 & 0 & 1 / 6 & 2 / 3 & 1 / 6 & 0 & 0 & 0 & 0 & 0 \\
0 & 0 & 0 & 1 / 6 & 2 / 3 & 1 / 6 & 0 & 0 & 0 & 0 \\
0 & 0 & 0 & 0 & 1 / 6 & 2 / 3 & 1 / 6 & 0 & 0 & 0 \\
0 & 0 & 0 & 0 & 0 & 1 / 6 & 2 / 3 & 1 / 6 & 0 & 0 \\
0 & 0 & 0 & 0 & 0 & 0 & 1 / 6 & 2 / 3 & 1 / 6 & 0 \\
0 & 0 & 0 & 0 & 0 & 0 & 0 & 1 / 6 & 2 / 3 & 1 / 6 \\
0 & 0 & 0 & 0 & 0 & 0 & 0 & 0 & 1 / 6 & 1 / 3
\end{array}\right]
$$

Previously it was shown that the differentiation matrix has the form,

$$
\mathcal{D}=b^{T} B^{-1} A\left(b^{T}\right)^{-1}
$$




\begin{tabular}{||c|c|c|c|c|c|c||}
\hline $\begin{array}{c}\text { Grid } \\
\text { Size }\end{array}$ & $\begin{array}{c}\text { Inner } \\
l_{1} \text { Error }\end{array}$ & $\begin{array}{c}\text { Error } \\
\text { Ratio }\end{array}$ & $\begin{array}{c}\text { Boundary } \\
l_{1} \text { Error }\end{array}$ & $\begin{array}{c}\text { Error } \\
\text { Ratio }\end{array}$ & $\begin{array}{c}\text { Total } \\
l_{1} \text { Error }\end{array}$ & $\begin{array}{c}\text { Error } \\
\text { Ratio }\end{array}$ \\
\hline 16 & $6.6 e^{-4}$ & & .025 & & .0049 & \\
\hline 32 & $3.4 e^{-5}$ & 19.4 & .013 & 1.9 & .0012 & 4.1 \\
\hline 64 & $2.1 e^{-6}$ & 16.2 & .0069 & 1.9 & $3.0 e^{-4}$ & 4.0 \\
\hline 128 & $1.3 e^{-7}$ & 16.2 & .0035 & 2.0 & $7.5 e^{-5}$ & 4.0 \\
\hline 256 & $8.3 e^{-9}$ & 15.7 & .0018 & 1.9 & $1.9 e^{-5}$ & 3.9 \\
\hline
\end{tabular}

Table 1: Calculated order of accuracy for a 1st order spline-based wavelet differentiation matrix for truncated B-spline boundary construction.

For $n=1$ the matrix $b$ is the identity.

\subsubsection{Computed Accuracy}

Again, one can estimate the order of accuracy by applying the above defined matrix $\mathcal{D}$ to evenly-spaced samples of the function

$$
f(x)=\frac{1}{2+\sin (x)},
$$

and comparing to the exact derivative.

In table (7.1.1) 'Inner Error' denotes the error at the middle $50 \%$ of the grid points, and the boundary error is the error at the two outermost grid points at each end of the interval. From the table it can be seen that the superconvergence is lost at the boundaries. That is, in the middle of the interval the 4 th-order superconvergence is obtained, whereas at the boundary the differentiation is only 1st-order accurate.

\subsection{Example for 3-rd Order Spline}

For the 3rd order B-splines the calculations are similarly straightforward. There is, however, one problem: the matrix $b$ is ill-conditioned. We need the inverse of $b$ and for grids larger than about 32 points the errors introduced by inverting $b$ make it very difficult to determine the accuracy. One can avoid this problem when testing accuracy by simply not including $b$ in the calculations. That is, $b$ comes from the quadrature equations and plays no role in the differencing. The following are the matrices $A$ and $B$ produced by the truncated B-spline boundary construction: 


$$
\begin{aligned}
& A= \\
& {\left[\begin{array}{cccccccccc}
-1 / 2 & -9 / 20 & 9 / 10 & 1 / 20 & 0 & 0 & 0 & 0 & 0 & 0 \\
-71 / 20 & -8 & 87 / 10 & 14 / 5 & 1 / 20 & 0 & 0 & 0 & 0 & 0 \\
-19 / 10 & -127 / 10 & -1 / 2 & 49 / 4 & 14 / 5 & 1 / 20 & 0 & 0 & 0 & 0 \\
-1 / 20 & -14 / 5 & -49 / 4 & 0 & 49 / 4 & 14 / 5 & 1 / 20 & 0 & 0 & 0 \\
0 & -1 / 20 & -14 / 5 & -49 / 4 & 0 & 49 / 4 & 14 / 5 & 1 / 20 & 0 & 0 \\
0 & 0 & -1 / 20 & -14 / 5 & -49 / 4 & 0 & 49 / 4 & 14 / 5 & 1 / 20 & 0 \\
0 & 0 & 0 & -1 / 20 & -14 / 5 & -49 / 4 & 0 & 49 / 4 & 14 / 5 & 1 / 20 \\
0 & 0 & 0 & 0 & -1 / 20 & -14 / 5 & -49 / 4 & 1 / 2 & 127 / 10 & 19 / 10 \\
0 & 0 & 0 & 0 & 0 & -1 / 20 & -14 / 5 & -87 / 10 & 8 & 71 / 20 \\
0 & 0 & 0 & 0 & 0 & 0 & -1 / 20 & -9 / 10 & 9 / 20 & 1 / 2
\end{array}\right]} \\
& B= \\
& {\left[\begin{array}{ccccccccc}
1 / 7 & 129 / 140 & 3 / 7 & 1 / 140 & 0 & 0 & 0 & 0 & 0 \\
129 / 140 & 302 / 35 & 531 / 70 & 6 / 7 & 1 / 140 & 0 & 0 & 0 & 0 \\
3 / 7 & 531 / 70 & 599 / 35 & 1191 / 140 & 6 / 7 & 1 / 140 & 0 & 0 & 0 \\
1 / 140 & 6 / 7 & 1191 / 140 & 604 / 35 & 1191 / 140 & 6 / 7 & 1 / 140 & 0 & 0 \\
0 & 1 / 140 & 6 / 7 & 1191 / 140 & 604 / 35 & 1191 / 140 & 6 / 7 & 1 / 140 & 0 \\
0 & 0 & 1 / 140 & 6 / 7 & 1191 / 140 & 604 / 35 & 1191 / 140 & 6 / 7 & 1 / 140 \\
0 & 0 & 0 & 1 / 140 & 6 / 7 & 1191 / 140 & 599 / 35 & 531 / 70 & 3 / 7 \\
0 & 0 & 0 & 0 & 1 / 140 & 6 / 7 & 531 / 70 & 302 / 35 & 129 / 140 \\
0 & 0 & 0 & 0 & 0 & 1 / 140 & 3 / 7 & 129 / 140 & 1 / 7
\end{array}\right]}
\end{aligned}
$$

\subsubsection{Computed Accuracy}

If we apply the matrix $\mathcal{D}=B^{-1} A$ to the evenly-spaced samples of the function

$$
f(x)=\frac{1}{2+\sin (\xi x)}
$$

for a fixed wave number $\xi$ it is difficult to determine the accuracy both at the boundaries and in the middle of the interval at the same time. That is, it was proven that the accuracy 'in the middle' is $2 n+2$ or 8 when $n=3$. For the current boundary construction, however, the accuracy is 3 at the boundaries. This vast difference in accuracy requires that the frequency of the test function be low in order to test the boundary accuracy and high in order to test the superconvergence in the middle of the interval.

In table (7.2.1) the wave number is $\xi=2 \pi$. This wave number is sufficiently high to test the superconvergence.

$$
\mathcal{D}=B^{-1} A
$$




\begin{tabular}{||c|c|c|c|c|c|c||}
\hline $\begin{array}{c}\text { Grid } \\
\text { Size }\end{array}$ & $\begin{array}{c}\text { Inner } \\
l_{1} \text { Error }\end{array}$ & $\begin{array}{c}\text { Error } \\
\text { Ratio }\end{array}$ & $\begin{array}{c}\text { Boundary } \\
l_{1} \text { Error }\end{array}$ & $\begin{array}{c}\text { Error } \\
\text { Ratio }\end{array}$ & $\begin{array}{c}\text { Total } \\
l_{1} \text { Error }\end{array}$ & $\begin{array}{c}\text { Error } \\
\text { Ratio }\end{array}$ \\
\hline 16 & $3.5 e^{-4}$ & & .022 & & .0037 & \\
\hline 32 & $1.8 e^{-7}$ & $1944=2^{10.9}$ & $6.6 e^{-4}$ & $33.3=2^{5.1}$ & $4.9 e^{-5}$ & $75.5=2^{6.2}$ \\
\hline 64 & $2.4 e^{-10}$ & $750=2^{9.5}$ & $3.5 e^{-5}$ & $18.9=2^{4.2}$ & $1.3 e^{-6}$ & $37.7=2^{5.2}$ \\
\hline 128 & $7.4 e^{-13}$ & $324=2^{8.3}$ & $2.8 e^{-6}$ & $12.5=2^{3.6}$ & $4.9 e^{-8}$ & $26.5=2^{4.7}$ \\
\hline 256 & $4.3 e^{-15}$ & $172=2^{7.4}$ & $3.1 e^{-7}$ & $9.0=2^{3.2}$ & $2.7 e^{-9}$ & $18.1=2^{4.2}$ \\
\hline
\end{tabular}

Table 2: Calculated order of accuracy for a 3rd order spline-based wavelet differentiation matrix for the truncated B-spline boundary construction. The test function has a high frequency in order to capture the 8 th order differentiation accuracy.

\begin{tabular}{||c|c|c|c|c|c|c||}
\hline $\begin{array}{c}\text { Grid } \\
\text { Size }\end{array}$ & $\begin{array}{c}\text { Inner } \\
l_{1} \text { Error }\end{array}$ & $\begin{array}{c}\text { Error } \\
\text { Ratio }\end{array}$ & $\begin{array}{c}\text { Boundary } \\
l_{1} \text { Error }\end{array}$ & $\begin{array}{c}\text { Error } \\
\text { Ratio }\end{array}$ & $\begin{array}{c}\text { Total } \\
l_{1} \text { Error }\end{array}$ & $\begin{array}{c}\text { Error } \\
\text { Ratio }\end{array}$ \\
\hline 16 & $1.6 e^{-8}$ & & $1.8 e^{-6}$ & & $2.7 e^{-7}$ & \\
\hline 32 & $4.1 e^{-11}$ & $390=2^{8.6}$ & $3.5 e^{-7}$ & $5.1=2^{2.4}$ & $2.3 e^{-8}$ & $11.7=2^{3.6}$ \\
\hline 64 & $8.9 e^{-15}$ & $4607=2^{12.2}$ & $5.1 e^{-8}$ & $6.9=2^{2.8}$ & $1.7 e^{-9}$ & $13.5=2^{3.8}$ \\
\hline 128 & $4.6 e^{-15}$ & & $6.8 e^{-9}$ & $7.5=2^{2.9}$ & $1.1 e^{-10}$ & \\
\hline 256 & $8.2 e^{-15}$ & & $8.8 e^{-10}$ & $7.7=2^{2.9}$ & $7.4 e^{-12}$ & \\
\hline 512 & $1.8 e^{-14}$ & & $1.1 e^{-10}$ & $8=2^{3}$ & $4.9 e^{-13}$ & \\
\hline
\end{tabular}

Table 3: Calculated order of accuracy for a 3rd order spline-based wavelet differentiation matrix for the truncated B-spline boundary construction. The test function has a low frequency in order to capture the 3rd order differentiation accuracy.

Now the lower frequency $\xi=1$ is used to test the accuracy at the boundaries. See table (7.2.1). Note that in table (7.2.1) that a few of the entries in the 'Error Ratio' columns are blank. This is done whenever the 'Inner Error' is of the order $10^{-15}$ and can no longer be resolved on the computer. Table (7.2.1) indicates the accuracy only at the boundaries. As in table (7.2.1) it can be seen that the boundary accuracy is 3rd order and, consequently, the superconvergence is lost at the boundaries.

The next section contains a few comments on this loss of superconvergence. 


\section{Loss of Superconvergence}

In [5] it was shown that under a given boundary construction the Daubechies-based wavelet superconvergence which was proved in [4] is lost. In this paper we have encountered a similar phenomenon. I believe that this in not simply a function of the the particular boundary functions which were chosen but a general characteristic for numerical methods which are based on approximation by translation. In fact, Gottlieb, et. al, see [3], have shown that the superconvergence encountered in the finite element method under periodic boundary conditions cannot be recovered when nonperiodic boundary conditions are present: '... no matter how the approximating subspace $S^{h}$ is chosen, the superconvergence property is lost if there are characteristics leaving the domain.'

Superconvergence is a property which depends on the overlap of bases functions with neighboring bases functions. At the boundaries the overlap only occurs on one side. For a Daubechies-based wavelet system where the bases functions are orthogonal there is a minimum amount of overlap which must occur at the boundary in order to maintain the superconvergence. That is, for a Daubechies 4 wavelet, 4-th order differentiation accuracy is encountered. In order to maintain the superconvergence one must have at least 3rd order differentiation accuracy at the boundaries. That is, four conditions must be satisfied requiring an overlap of four basis functions at the boundary. As of now, there are no boundary constructions satisfying this criterion.

For spline-based wavelet systems the situation is similar but slightly more complicated since the underlying functions, B-splines, are not orthogonal. Again, however, a general rule-of-thumb is that the overlap of the B-splines at the boundary should be the same as the superconvergence encountered with periodic boundary conditions. That is, for the 1st order B-spline we have shown here that the differentiation is 4 th order accurate where periodic boundary conditions are assumed. This would require an overlap of 4 boundary functions in order to have 3rd order boundary accuracy 
which maintains the 4 th order overall accuracy.

The next section concludes this paper. 


\section{Conclusion}

Let me restate a few of the salient features of this paper. First of all, circularity plays a prominent role when periodic boundary conditions are assumed. The benefit of working with circular matrices is that they commute. In the periodic case this implies that the differentiation matrix is the same as the derivative projection matrix,

$$
\mathcal{D}=C^{-1} D C=D .
$$

The proof of superconvergence is, however, the most meaningful result of the paper. I have shown that the matrix $D$ displays accuracy of order $2 n+2$ where $n$ is the order of the underlying B-spline used to construct the scaling functions. Also, it was seen that this accuracy holds regardless of of whether the scaling functions are chosen to be orthogonal under translation or not. That is, the scaling functions can be required to be $\mathrm{B}$-splines, $\mathrm{C}$-splines, or orthogonal. The parameters which dictate the properties of the scaling function 'divide out' when the differentiation matrix is calculated. Because of this feature, only one theory is necessary to illustrate the accuracy for the spline-based differentiation matrix when periodic boundary conditions are assumed.

When the boundary conditions are, on the other hand, not periodic it has been shown that for boundary functions constructed from truncated B-splines that the superconvergence is lost at the boundary. I speculate that this loss of superconvergence will continue to be a characteristic of wavelet bases defined on an interval.

It is, also, interesting to note the relationship between spline-based wavelet methods and compact finite difference methods. That is, the underlying character of a spline-based wavelet method can be understood in terms of compact finite difference schemes. 


\section{References}

[1] I. Daubechies, "Orthonormal Basis of Compactly Supported Wavelets", Comm. Pure Appl. Math., 41 (1988), pp. 909-996.

[2] P. Davis, "Circulant Matrices", Wiley-Interscience, 1979.

[3] David Gottlieb, Bertil Gustafsson, Pelle Olsson, and Bo Strand, "On the Superconvergence of Galerkin Methods for Hyperbolic IBVP", July 29, 1993, submitted to SIAM J. Sci. Comp.

[4] L. Jameson, "On The Wavelet Based Differentiation Matrix", Journal of Scientific Computing, September 1993.

[5] L. Jameson, "Daubechies-Based Wavelets on an Interval", to be submitted to SIAM J. Sci. Comp.

[6] J. Liandrat and P. Tchamitchian, "Resolution of the 1D Regularized Burgers Equation using a Spatial Wavelet Approximation Algorithm and Numerical Results", ICASE Report No. 90-83 (1990).

[7] I. J. Schoenberg, "Contribution to the problem of approximation of equidistant data by analytic functions", Quart. Appl. Math. 4 (1946), 45-99, 112-141.

[8] I. J. Schoenberg, "Cardinal Spline Interpolation", CBMS-NSF series in Applied Math 12, SIAM Publ., Philadelphia, PA, 1973.

[9] G. Strang, "Wavelets and Dilation Equations: A Brief Introduction", SIAM Review, Vol. 31, No.4, pp. 614-627, Dec. 1989.

[10] M. Unser and A. Aldroubi, "Polynomial Splines and Wavelets- A Signal Processing Perspective", Wavelets- A Tutorial in Theory and Applications, C. K. Chui (ed.) pp. 91-112 (1992), Academic Press, Inc. 
[11] M. Unser, A. Aldroubi, and M. Eden, "The $L_{2}$ polynomial spline pyramid: a discrete multiresolution representation of continuous signals", IEEE Pattern Anal. Mach. Intell., to appear.

[12] Wei Cai and JianZhong Wang, "Adaptive Wavelet Collocation Methods for Initial Value Boundary Problems of Nonlinear PDE's", ICASE Report No.93-48 NASA CR-191507, and submitted to SIAM J.Num. Anal. 



\section{REPORT DOCUMENTATION PAGE}

Form Approved

OMB No. 0704-0188

Public reporting burden for this collection of information is estimated to average $I$ hour per response, including the time for reviewing instructions, searching existing data sources, gathering and maintaining the data needed, and completing and reviewing the collection of information. Send comments regarding this burden estimate or any ather aspect of this collection of information, including suggestions for reducing this burden, to Washington Headquarters Services, Directorate or in

\begin{tabular}{|l|l|l}
\hline 1. AGENCY USE ONLY(Leave blank) & $\begin{array}{l}\text { 2. REPORT DATE } \\
\text { 3. REPORT TYPE AND DATES COVEREO } \\
\text { Contractor Report }\end{array}$
\end{tabular}

\begin{tabular}{|l|l|l|} 
& November 1993 & Contractor Report \\
\hline
\end{tabular}

4. TITLE AND SUBTITLE

ON THE SPLINE-BASED WAVELET

DIFFERENTIATION MATRIX

5. FUNDING NUMBERS

C NAS1-19480

WU 505-90-52-01

\section{AUTHOR(S)}

Leland Jameson

7. PERFORMING ORGANIZATION NAME(S) AND ADDRESS(ES)

Institute for Computer Applications in Science

and Engineering

Mail Stop 132C, NASA Langley Research Center

REPORT NUMBER

Hampton, VA 23681-0001

9. SPONSORING/MONITORING AGENCY NAME(S) AND ADDRESS(ES)

National Aeronautics and Space Administration

Langley Research Center

Hampton, VA 23681-0001

ICASE Report No. $93-80$

11. SUPPLEMENTARY NOTES

Langley Technical Monitor: Michael F. Card

Final Report

To be submitted to SIAM Journal on Numerical Analysis

12a. DISTRIBUTION/AVAILABILITY STATEMENT

12b. DISTRIBUTION CODE

Unclassified-Unlimited

Subject Category 64

13. ABSTRACT (Maximum 200 words)

The differentiation matrix for a spline-based wavelet basis will be constructed. Given an n-th order spline basis it will be proven that the differentiation matrix is accurate of order $2 n+2$ when periodic boundary conditions are assumed. This high accuracy, or superconvergence, is lost when the boundary conditions are no longer periodic. Furthermore, it will be shown that spline-based bases generate a class of compact finite difference schemes.

14. SUBJECT TERMS

differentiation matrix; wavelets; superconvergence
10. SPONSORING/MONITORING AGENCY REPORT NUMBER

NASA CR-191557

ICASE Report No. $93-80$

\begin{tabular}{|l|l|}
\hline 17. SECURITY CLASSIFICATION & 18. SECURITY CLASSIFICATION \\
OF REPORT & $\begin{array}{l}\text { OF THIS PAGE } \\
\text { Unclassified }\end{array}$ \\
Unclassified & \\
\hline NSN 7540-01-280-5500 &
\end{tabular}

HU.S. GOVERNMENT PRINTING OFTICE: 1993 - 528-064/86036
15. NUMBER OF PAGES

37

16. PRICE CODE

$\mathrm{A} 03$

19. SECURITY CLASSIFICATION OF ABSTRACT

20. LIMITATION OF ABSTRACT

Standard form 298(Rev. 2-89) Prescribed by ANSI Std Z39-18 298-102 


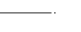

